Original Research Paper

\title{
One Real-Time Fluorescent Loop-Mediated Isothermal Amplification Combined with Propidium Monoazide for Detection of Viable Vibrio Parahaemolyticus in Seafood
}

\author{
${ }^{1}$ Huanteng Yu, ${ }^{1}$ Jiehong Fang, ${ }^{2}$ Biao Ma, ${ }^{1}$ Jiali Li and ${ }^{1,2}$ Mingzhou Zhang \\ ${ }^{1}$ China Jiliang University, Hangzhou, China \\ ${ }^{2}$ Zhejiang Provincial Key Laboratory of Biometrology and Inspection and Quarantine, \\ China Jiliang University, Hangzhou, China
}

Article history

Received: 01-04-2019

Revised: 30-04-2019

Accepted: 25-06-2019

Corresponding Author: Mingzhou Zhang

Zhejiang Provincial Key

Laboratory of Biometrology and Inspection and Quarantine, China Jiliang University,

Hangzhou, China

E-mail: zmzcjlu@cjlu.edu.cn

\begin{abstract}
Vibrio parahaemolyticus is one of the crucial foodborne pathogens in the world. At present, the rapid detection methods for $V$. parahaemolyticus cannot distinguish between dead and viable cells and false positive results may occur. In this study, one rapid and accurate method that combines Propidium Monoazide (PMA) with real-time fluorescent loop-mediated isothermal amplification (LAMP) using SYTO16 dye was developed to detect viable cells of $V$. parahaemolyticus. LAMP amplification was performed on specific primers designed for tox $R$ gene of $V$. parahaemolyticus and the concentration of PMA and the maximum concentration of dead cells were optimized. The results showed that the concentration of PMA was $10 \mu \mathrm{M}$ and PMA could efficiently treat dead cells up to $4.5 \times 10^{5} \mathrm{CFU} / \mathrm{mL}$. The detection sensitivity of real-time fluorescent PMA-LAMP were $4.5 \times 10^{\circ} \mathrm{CFU} / \mathrm{mL}$ and PMA-qPCR were $4.5 \times 10^{1} \mathrm{CFU} / \mathrm{mL}$. In addition, the correlation coefficients $\left(\mathrm{R}^{2}\right)$ is 0.9992 , indicating that SYTO-16 dyed real-time fluorescent PMA-LAMP assay can be used for quantification with high sensitivity. This method exhibits high specificity and sensitivity, can be used as an effective tool for rapid detection of $V$. parahaemolyticus and a scientific basis to follow the effect of the pathogen infection on growth of cultured seafood.
\end{abstract}

Keywords: $V$. parahaemolyticus, Propidium Monoazide, Real-Time Fluorescent Loop-Mediated Isothermal Amplification, Seafood

\section{Introduction}

Vibrio parahaemolyticus, a kind of halophilic Gramnegative bacterium, has been considered as the leading causes of seafood-borne illness in the world (Nair et al., 2007; Guo et al., 2010). V. parahaemolyticus was first isolated from a food poisoning incident in Osaka and was generally widely distributed in areas such as coastal areas or estuary of rivers ( $\mathrm{Su}$ and Liu, 2007). It is reported that seafood infected with $V$. parahaemolyticus can lead to nausea, vomiting, diarrhea, abdominal cramps, low fever, chills and even gastroenteritis and septicemia (Zhong et al., 2016). In addition, the Vibrio species have been highly resistant to most commercially available antibiotics (Stalin and Srinivasan, 2016). Therefore, rapid and sensitive detection of $V$. parahaemolyticus is significant to prevent the disease.

Most traditional biochemical culture detection methods, including detection of $V$. parahaemolyticus by the gold standard method Bacteriological Analytical Manual (BAM), are time-consuming and laborious, which are not conducive to timely identification of pathogens, elimination of safety hazards or prompt treatment (Zhi et al., 2017). In recent years, molecular diagnostic techniques have been developed for $V$. parahaemolyticus detection, such as PCR and real-time PCR, but both of which require complicated instruments and professionals (Davis et al., 2004; Cai et al., 2006; Bunpa et al., 2018; Xu et al., 2018). These drawbacks can be compensated by using Loop-Mediated Isothermal Amplification (LAMP) method, which can utilize $B s t$ DNA polymerase and a set of four primers targeted six distinct regions for efficient amplification of the target gene under isothermal condition $\left(60-65{ }^{\circ} \mathrm{C}\right)$ within 60 min (Notomi et al., 2000). Additionally, loop primers could be added to the assay reaction which was designed 
according to the four primers set to enhance efficiency and increase specificity of the assay (Nagamine et al., 2002). Moreover, the quantitative detection of LAMP products can be realized in real time by using fluorescent dyes and evaluating the amplification curve of fluorescent signal (Oscorbin et al., 2016). In our study, fluorescence dye SYTO-16 was used to visualize the LAMP assay with excitation wavelength of $488 \mathrm{~nm}$ and emission wavelength of $518 \mathrm{~nm}$. SYTO-16 is a kind of cell permeant nucleic acid stain that shows a large fluorescence enhancement upon binding nucleic acids (Udovich et al., 2010).

However, none of the above methods can distinguish between dead and viable cells, which often result in false positive results. Ethidium Bromide Monoazide (EMA) and propidium monoazide (PMA) are often applied to eliminate the interference of dead cells (Wang and Mustapha, 2010; Zhu et al., 2012; Saiyudthong and Trevanich, 2013). PMA was less toxic than EMA and had higher specificity to dead cells (Fittipaldi et al., 2012).

In this study, we report the use of SYTO-16 dyed real-time LAMP with six target-specific primers for the detection of $V$. parahaemolyticus in seafood samples. We also used PMA treatment prior to realtime LAMP screening to quantitatively detect viable V. parahaemolyticus.

\section{Materials and Methods}

\section{Preparation of Bacterial Strains}

V. parahaemolyticus ATCC 17082 was used for realtime fluorescent PMA-LAMP assay optimization and sensitivity test. Two additional $V$. parahaemolyticus strains (H4-3 and FJ14A) isolated from seafood and a total of 12 non- $V$. parahaemolyticus strains including Vibrio cholerae, Salmonella enteritidis, Staphylococcus aureus, Escherichia coli, Listeria monocytogenes, Shigella, Staphylococcus epidermidis, Vibrio hollisae, Vibrio mimicus, Vibrio damsela, Vibrio vulnificus, Vibrio fluvialis were employed for the specificity test (Table 1). $25 \mathrm{~g}$ of seafood samples were dissolved in $225 \mathrm{~mL} 3 \%$ Nacl APW. V. parahaemolyticus strains were cultured 18 $\mathrm{h}$ on TCBS agar plate (Hopebio, China) at $37{ }^{\circ} \mathrm{C}$, showing a round, opaque, green or bluish colony on TCBS agar plate with a diameter of 2-3 mm. A single colony was transferred to alkaline saline peptone water of $3 \%$ sodium chloride and grown at $37^{\circ} \mathrm{C}$ overnight. The bacterial culture was used for the extraction of genome, PMA treatment or plate counting. The non- $V$. parahaemolyticus strains were cultured in Luria-Bertani medium (LB, Sangon, China) at $37^{\circ} \mathrm{C}$ overnight. All experiments with bacteria culture were carried out in a biosafety level 2 laboratory.

\section{Preparation of Dna Template}

The DNA template of Gram-negative bacteria, including $V$. parahaemolyticus was prepared by the method described previously (Zhi et al., 2017; Fang et al., 2018). The bacteria cells were collected by centrifugation at $10000 \times g$ for $5 \mathrm{~min}$. The pellets were washed and resuspended in $50 \mu \mathrm{L}$ Tris-EDTA buffer (10 mM Tris-HCl, 1 mM EDTA, pH 8.0). The suspension was frozen at $-20{ }^{\circ} \mathrm{C}$ for $10 \mathrm{~min}$ and heated immediately at $100{ }^{\circ} \mathrm{C}$ for $10 \mathrm{~min}$. After centrifugation at $12000 \times g$ for $5 \mathrm{~min}$, the supernatant containing bacteria DNA was used as the DNA template. The DNA template of Gram-positive bacteria, spiked samples and practical samples were extracted by a bacterial DNA extraction kit according to the instruction (Sangon, China).

Table 1: Information of bacterial strains used for specificity tests in the study

\begin{tabular}{|c|c|c|c|}
\hline Species & ID of strains & Result of LAMP & Result of qPCR \\
\hline \multirow[t]{3}{*}{ Vibrio parahaemolyticus } & ATCC 17802 & + & + \\
\hline & $\mathrm{H} 4-3^{\mathrm{b}}$ & + & + \\
\hline & FJ14A ${ }^{b}$ & + & + \\
\hline Vibrio cholerae & GIMCC 1.449 & - & - \\
\hline Salmonella enteritidis & GIMCC 1.345 & - & - \\
\hline Staphylococcus aureus & GIMCC 1.142 & - & - \\
\hline Escherichia coli & GIMCC 1.201 & - & - \\
\hline Listeria monocytogenes & ATCC 19115 & - & - \\
\hline Shigella & GIMCC 1.424 & - & - \\
\hline Staphylococcus epidermidis ${ }^{a}$ & 1 & - & - \\
\hline Vibrio hollisae ${ }^{a}$ & l & - & - \\
\hline Vibrio mimicus $^{a}$ & / & - & - \\
\hline Vibrio damsela ${ }^{a}$ & l & - & - \\
\hline Vibrio vulnificus ${ }^{a}$ & / & - & - \\
\hline Vibrio fluvialis $^{a}$ & l & - & - \\
\hline
\end{tabular}

GIMCC: Guangdong Microbiology Culture Center, ATCC: American Type Culture Collection

${ }^{a}$ Afforded by Zhoushan Entry-Exit Inspection and Quarantine Bureau without marks

${ }^{\mathrm{b}}$ Afforded by Zhoushan Entry-Exit Inspection and Quarantine Bureau with marks 
Table 2: Sequences of Vibrio parahaemolyticus LAMP primers and qPCR primers/probe

\begin{tabular}{|c|c|c|c|}
\hline Primers & Sequence (5'-3') & Target gene & $\begin{array}{l}\text { Fragment } \\
\text { length (bp) }\end{array}$ \\
\hline & LAMP Primers & $\operatorname{tox} R$ & 192 \\
\hline VP -F3 & TAATTCGCTCGCAGACCA & & \\
\hline VP -B3 & CGCAGAGTAGAAATCGCT & & \\
\hline VP -FIP & TCTCCGCCAACATCATTTTGGAGTAGGCAACGAAGTTGTACGA & & \\
\hline VP -BIP & TAACCCGTAACGTTTTAGCTTCACGTCAGGCTTGAGTCATCCA & & \\
\hline VP -LF & ACGGCTTTCGTTGCTTCC & & \\
\hline \multirow[t]{2}{*}{ VP -LB } & TGTTTGGCGTGAGCAAGG & & \\
\hline & qPCR Primers & & 213 \\
\hline VP -F & GTGCAGGTTTATGTGTTC & & \\
\hline VP -R & CCTGAATACGGGATTAGAC & & \\
\hline VP-Probe & FAM-CGCTGATGGCACAGATCACAG-TAMRA & & \\
\hline
\end{tabular}

$\mathrm{F}$ refers to forward and $\mathrm{R}$ refers to reverse

\section{PMA Treatments and Optimization of PMA Working Concentration}

To obtain the optimal concentration of PMA treatment, dead cells of $V$. parahaemolyticus ATCC 17082 were prepared by boiling at $95{ }^{\circ} \mathrm{C}$ for $15 \mathrm{~min}$ according to the method described previously (Zhi et al., 2017; Fang et al., 2018). To confirm all the bacteria were killed, the cells after treated were cultured on TCBS at $37{ }^{\circ} \mathrm{C}$ for $48 \mathrm{~h}$. Then $20 \mathrm{mM}$ PMA (Biotium Inc., Hayward, CA, USA) was added at final concentration of $0,5,10,15,20,40$ and 80 $\mu \mathrm{M}$ to $500 \mu \mathrm{L}$ suspension containing $4.5 \times 10^{5} \mathrm{CFU} / \mathrm{mL}$ dead $V$. parahaemolyticus ATCC 17082 in $1.5 \mathrm{~mL}$ eppendorf tubes and solution was mixed up and down for several times to make the sample fully in contact with the PMA dye and left in the dark for $5 \mathrm{~min}$. Then the tubes placed on ice were transferred to $60 \mathrm{~W}$ PMA-Lite Photolysis Device (Biotium) and were illuminated for $30 \mathrm{~min}$ by LED lights to activate the PMA. In order to obtain better concentration of dead cells, the copy number of dead cells to be processed was set as $4.5 \times 10^{8}, 4.5 \times 10^{7}, 4.5 \times 10^{6}, 4.5 \times 10^{5}$, $4.5 \times 10^{4} \mathrm{CFU} / \mathrm{mL}$. Three control groups were set at the same time, which were $4.5 \times 10^{5} \mathrm{CFU} / \mathrm{mL}$ pure viable cells that were not treated by PMA, pure dead cells treated by PMA and negative control. The results were determined by whether the DNA amplification of dead cells could be completely suppressed.

\section{LAMP Primers and Establishment of Real-time Fluorescent LAMP Assay}

The sequence of tox $R$ gene were obtained from GenBank in NCBI database (accession numbers: GQ228073.1). The specific regions were selected as the target fragment after aligning by using Clustal W software. Based on the detailed analysis and comparison, specific primers (Table 2) of LAMP including two loopprimers were designed with the online tool Primer Explorer V5.0 (http://primerexplorer.jp/e/), which was supplied by Eiken Chemical (Tokyo, Japan). The DNA template of $2.5 \mu \mathrm{L}$ was added to the amplification reagent (total volume $25 \mu \mathrm{L}$ ) containing $0.4 \mu \mathrm{M}$ outer primer F3 and B3, 3.2 $\mu \mathrm{M}$ inner primer FIP and BIP, 1.6 $\mu \mathrm{M}$ loop primer $\mathrm{LF}$ and $\mathrm{LB}$, together with $20 \mathrm{mM}$ Tris- $\mathrm{HCl}(\mathrm{pH} 8.8)$, $10 \mathrm{mM} \mathrm{KCl}, 8 \mathrm{mM} \mathrm{MgSO} 4,10 \mathrm{mM}\left(\mathrm{NH}_{4}\right)_{2} \mathrm{SO}_{4}, 0.1 \%$ Tween20 (Sigma-Aldrich), $0.8 \mathrm{M}$ betaine, $0.5 \mathrm{mM} \mathrm{MnCl}_{2}$, $1.4 \mathrm{mM}$ dNTPs (Thermo Scientific, USA), $10 \mu \mathrm{M}$ SYTO16 (Thermo Scientific, USA) and $0.32 \mathrm{U} / \mu \mathrm{L}$ Bst 2.0 DNA polymerase (NEB, USA). No-template control reactions contained distilled water instead of template DNA. The amplification program was: $65^{\circ} \mathrm{C}$ for $1 \mathrm{~min}$, followed by 40 cycles of $65^{\circ} \mathrm{C}$ for $60 \mathrm{sec}$. Fluorescence signals were collected using the SYBR channel by using the ABI Step One Plus ${ }^{\mathrm{TM}}$. Fluorescence data were analyzed after amplification using the ABI software.

\section{qPCR Primers and Establishment of qPCR Assay}

A TaqMan-based qPCR assay was also established by targeting the tox $R$ gene. Primers and the TaqMan probe were designed by Beacon Designer 7.9 (Table 2) and were synthesized by Invitrogen Biotechnology Co. Ltd. The reaction mix contained $2.5 \mu \mathrm{L}$ DNA template, 0.2 $\mu \mathrm{L}$ RoxII, $0.8 \mu \mathrm{M}$ of primer sets, $0.1 \mu \mathrm{M}$ of probe and 10 $\mu \mathrm{L} 2 \times$ Premix Ex Taq (Takara, Japan) and $\mathrm{ddH}_{2} \mathrm{O}$ to a final volume of $20 \mu \mathrm{L}$. The thermal cycle program was: $95{ }^{\circ} \mathrm{C}$ for $5 \mathrm{~min}$, followed by 40 cycles of $95^{\circ} \mathrm{C}$ for 10 sec and $60{ }^{\circ} \mathrm{C}$ for $30 \mathrm{sec}$.

\section{Specificity and Sensitivity of Real-time Fluorescent PMA-LAMP and PMA-qPCR Assays}

To determine assay specificity, three viable and three heat-killed dead $V$. parahaemolyticus SSS strains and 12 non- $V$. parahaemolyticus strains including Vibrio cholerae, Salmonella enteritidis, Staphylococcus aureus, Escherichia coli, Listeria monocytogenes, Shigella, Staphylococcus epidermidis, Vibrio hollisae, Vibrio mimicus, Vibrio damsela, Vibrio vulnificus, Vibrio fluvialis (Table 1) were treated with PMA and DNA was extracted. 
To determine assay sensitivity, $V$. parahaemolyticus ATCC 17082 was grown to the mid-exponential growth phase and serially diluted 10 -fold in alkaline saline peptone water of $3 \%$ sodium chloride. The initial cell number was quantified by plate counting on plate count agar (Hopebio, China) using BAM methods. Concentrations of viable $V$. parahaemolyticus ATCC 17082 between $4.5 \times 10^{8}$ and $4.5 \times 10^{0} \mathrm{CFU} / \mathrm{mL}$ mixed with $4.5 \times 10^{5} \mathrm{CFU} / \mathrm{mL}$ dead V. parahaemolyticus ATCC 17082 cells were treated with the optimal concentration of PMA. Finally, DNA was extracted for sensitivity testing. Both tests were repeated three times. The amplification performance of real-time fluorescent PMA-LAMP was compared with real-time PMA-PCR. The threshold time was plotted against the log values of the detected molecules to generate an amplification standard curve.

\section{Evaluation of Real-Time Fluorescence PMA-LAMP Assay with Spiked Samples}

Fresh shrimp, sleevefish and cod samples were purchased from the local market and confirmed to be negative for $V$. parahaemolyticus using BAM methods. Each sample weighed $50 \mathrm{~g}$, homogenized and was added to $450 \mathrm{~mL}$ of alkaline saline peptone water of $3 \%$ sodium chloride under sterile conditions. The homogenates were contaminated with $V$. parahaemolyticus suspension which containing $4.5 \times 10^{5}$ to $4.5 \times 10^{0} \quad \mathrm{CFU} / \mathrm{mL}$ viable $V$. parahaemolyticus ATCC 17082 cells and $4.5 \times 10^{5}$ $\mathrm{CFU} / \mathrm{mL}$ dead cells. DNA extraction after PMA treatment were performed as the above description. Each sample was analyzed for the presence of $V$. parahaemolyticus using the real-time fluorescence PMA-LAMP, PMAqPCR and BAM methods.

\section{Evaluation of Real-Time Fluorescence PMA-LAMP Assay with Practical Samples}

A total of 139 practical samples (shrimp, sleevefish, trichiurus lepturus, cod, meretrix, grilled fish, grilled yellow croaker and dried squid) which purchased from local markets and afforded by Zhoushan Entry-exit Inspection and Quarantine Bureau were detected by the SYTO16-dyed real-time fluorescence PMA-LAMP and PMA-qPCR assays. After a pre-enrichment step at $37^{\circ} \mathrm{C}$ for $4 \mathrm{~h}, 2000 \times \mathrm{g}$, $50 \mathrm{~g}$ of each samples were diluted 1000-fold in alkaline saline peptone water of $3 \%$ sodium chloride and treated with PMA. DNA was extracted for realtime fluorescence PMA-LAMP and PMA-qPCR assays, with BAM method used as control.

\section{Data analysis}

Data collected from the real-time fluorescence PMALAMP and PMA-qPCR assays including standard curves were analyzed by ABI Step One Plus ${ }^{\mathrm{TM}}$ and Microsoft
Excel software (Microsoft Inc., USA). Results of qLAMP and qPCR were judged as positive ones when the $C_{t}$ value $\leq 35$. The coefficients of variation $(\mathrm{CV})$ of $\mathrm{Ct}$ values of the sensitivity and artificial contamination testing were statistically analyzed for the repeatability (CV value should be less than 10). In addition, the detection rate of PMA-LAMP, PMA-qPCR assay and BAM method for tests of practical samples was used to compare the coincidence rate between the three methods.

\section{Results}

The proper concentration of PMA working concentration and the maximum treatable concentration of dead $V$. parahaemolyticus cells

PMA, a kind of DNA binding dye, was applied prior to the DNA amplification assay to differentiate viable cells form dead cells by LED light treatment. The proper concentration is important that not only the amplification of dead cell DNA cannot be completely suppressed, but also had no toxicity to viable cells (Zhi et al., 2017; Fang et al., 2018). To obtain the proper concentration of PMA, an amount of $4.5 \times 10^{5} \mathrm{CFU} / \mathrm{mL}$ heat-killed $V$. parahaemolyticus dead cells or viable cells were treated with different concentration of PMA from 0 to $80 \mu \mathrm{M}$ (Fig. 1). The amplification of dead cells was suppressed fully by 10 $\mu \mathrm{M}$ to $80 \mu \mathrm{M}$ PMA treatment. In the meanwhile, when the concentration of PMA is $15 \mu \mathrm{M}$ or more, the amplification of viable cells was suppressed somewhat. Therefore, $10 \mu \mathrm{M}$ was considered to be the proper concentration of PMA.

The heat-killed $V$. parahaemolyticus dead cells were serially diluted from $4.5 \times 10^{8} \mathrm{CFU} / \mathrm{mL}$ to $4.5 \times 10^{4}$ CFU/mL. DNA was extracted for real-time fluorescent PMA-LAMP and PMA-qPCR after treated with $10 \mu \mathrm{M}$ PMA. The results indicated that the optimal $10 \mu \mathrm{M}$ PMA was effective for suppression $V$. parahaemolyticus dead cells lower than $4.5 \times 10^{5} \mathrm{CFU} / \mathrm{mL}$ (Fig. 2). The results is consistent with the previous report (Zhi et al., 2017; Fang et al., 2018).

\section{Specificity of Real-Time Fluorescent PMA-LAMP and PMA-qPCR}

The specificity of the real-time fluorescent PMALAMP and PMA-qPCR assay was determined using three viable $V$. parahaemolyticus cells, three heatkilled dead $V$. parahaemolyticus cells and 12 non- $V$. parahaemolyticus strains including Vibrio cholerae, Salmonella enteritidis, Staphylococcus aureus, Escherichia coli, Listeria monocytogenes, Shigella, Staphylococcus epidermidis, Vibrio hollisae, Vibrio mimicus, Vibrio damsela, Vibrio vulnificus, Vibrio fluvialis. The two developed methods were performed specifically that only three viable $V$. parahaemolyticus cells were observed to show positive results (Fig. 3). 


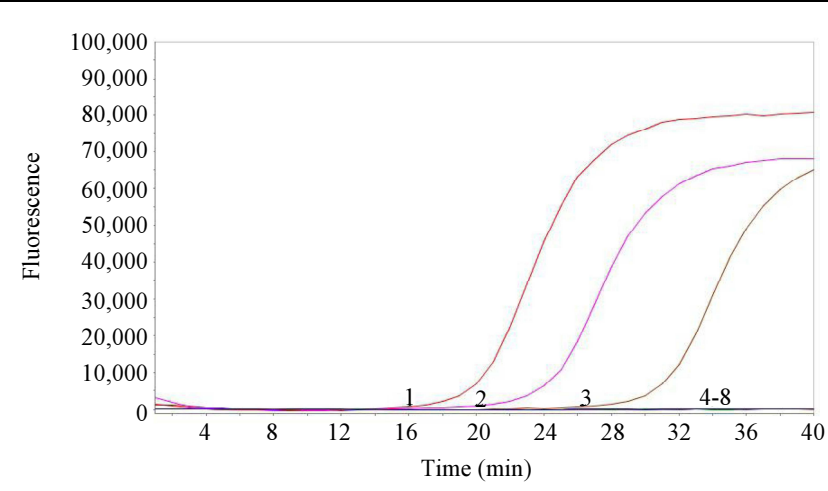

(a)

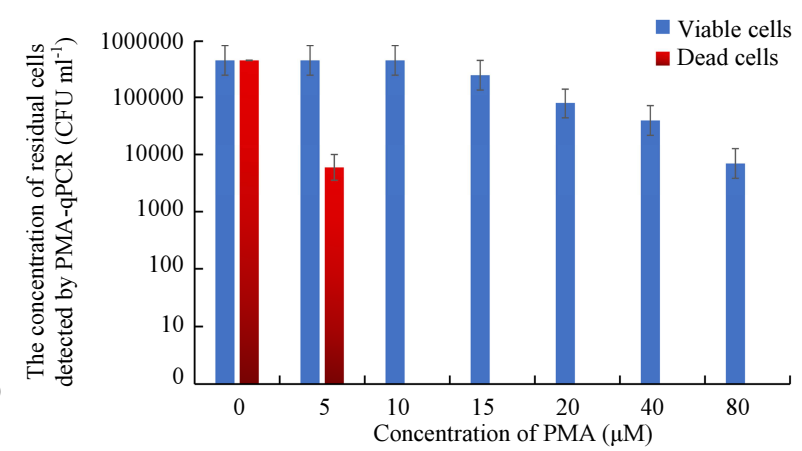

(b)

Fig. 1: The optimal concentration of propidium monoazide (PMA) for viable $V$. parahaemolyticus detection. (a) Heat-killed $V$. parahaemolyticus cells treated with different PMA concentrations and analysed by real-time fluorescent LAMP assay. 1: positive control (untreated $V$. parahaemolyticus); 2-8: $V$. parahaemolyticus treated with $0,5,10,15,20,40$ and $80 \mu \mathrm{M}$ PMA.

(b) Concentration of residual heat-killed and viable $V$. parahaemolyticus cells detected by qPCR after PMA treatment

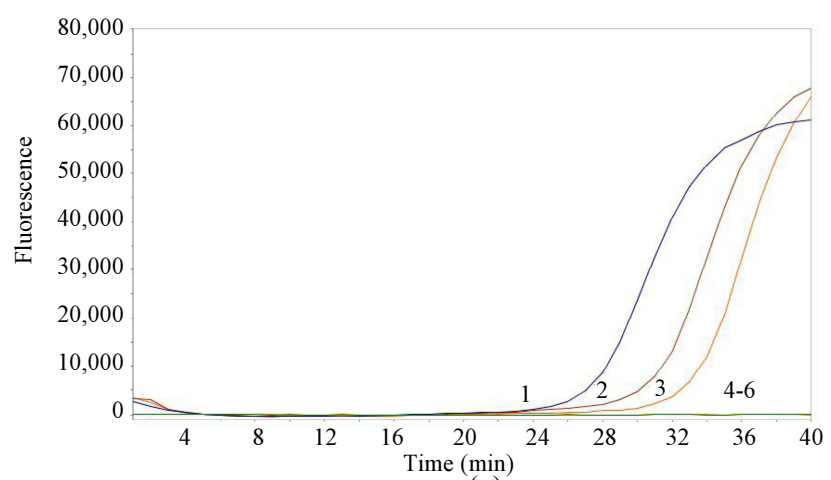

(a)

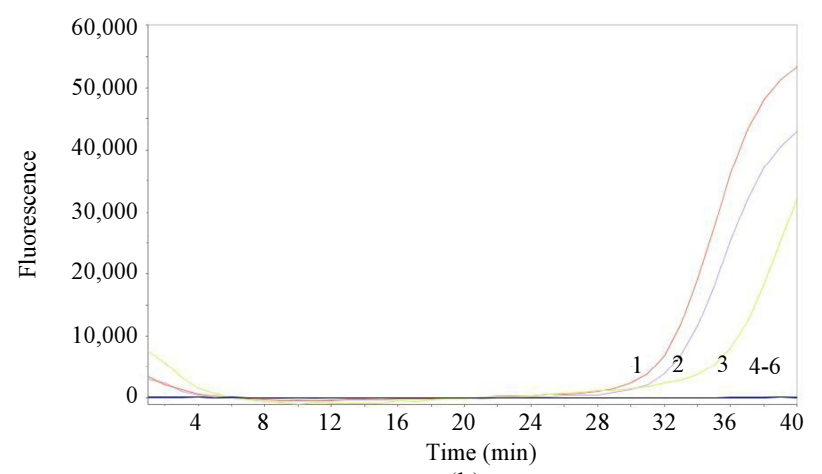

(b)

Fig. 2: Maximum heat-killed dead cell concentrations for propidium monoazide (PMA) treatment. (a) real-time fluorescent LAMP assay results. (b) real-time qPCR assay results. 1-5: $4.5 \times 10^{8}, 4.5 \times 10^{7}, 4.5 \times 10^{6}, 4.5 \times 10^{5}, 4.5 \times 10^{4} \mathrm{CFU} / \mathrm{mL}$; 6 : No template control

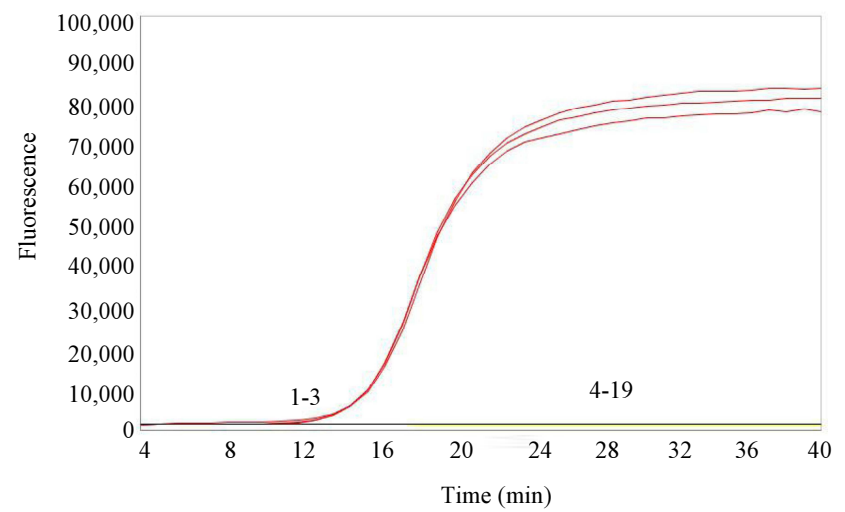

(a)

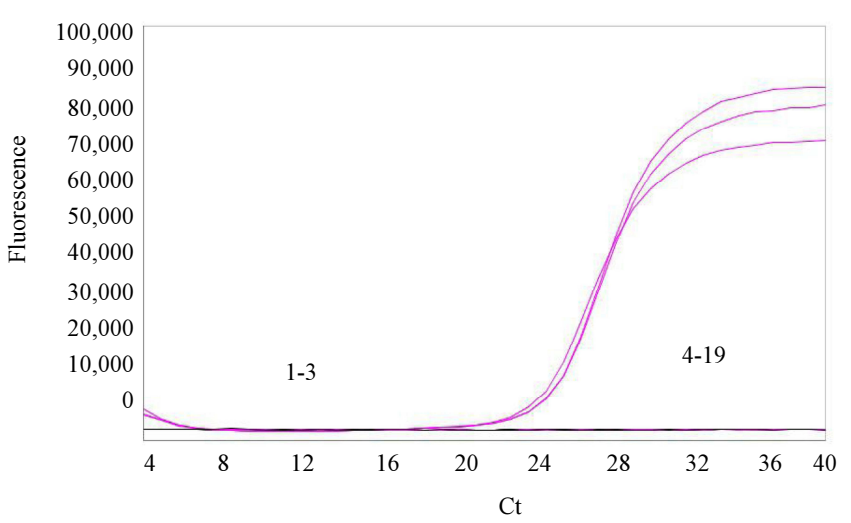

(b)

Fig. 3: Specificity of the real-time fluorescent PMA-LAMP and PMA-qPCR detection. Three standard strains of $V$. parahaemolyticus and 12 non- $V$. parahaemolyticus strains were amplified by real-time fluorescent PMA-LAMP assay (a) and PMA-qPCR assay (b). 1-3: V. parahaemolyticus positive strain, 4-6: heat-killed V. parahaemolyticus cells, 7-18: Vibrio cholerae, Salmonella enteritidis, Staphylococcus aureus, Escherichia coli, Listeria monocytogenes, Shigella, Staphylococcus epidermidis, Vibrio hollisae, Vibrio mimicus, Vibrio damsela, Vibrio vulnificus, Vibrio fluvialis, 19: No template control 


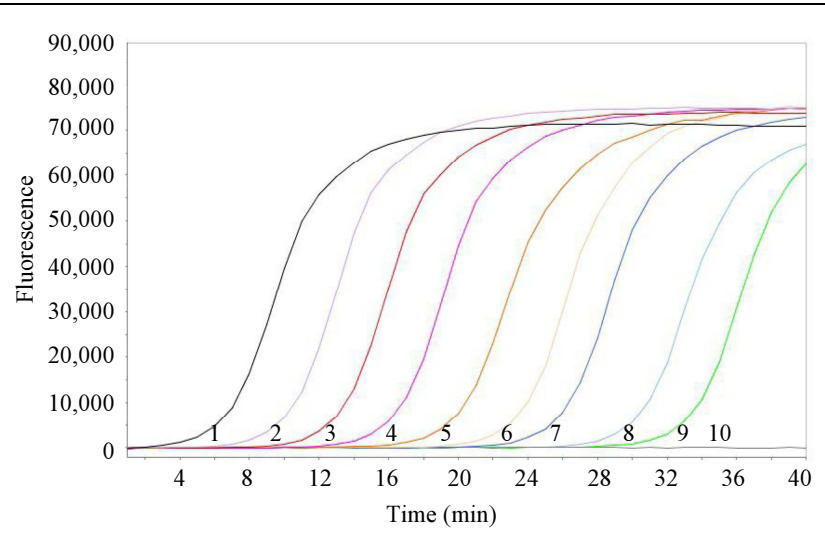

(a)

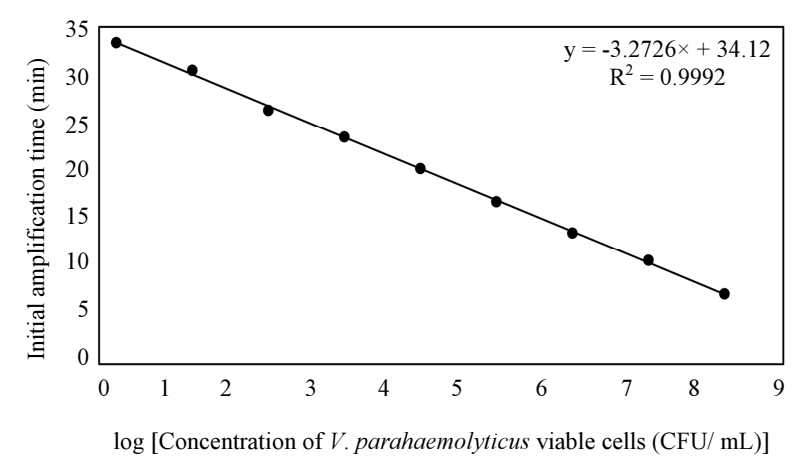

(c)

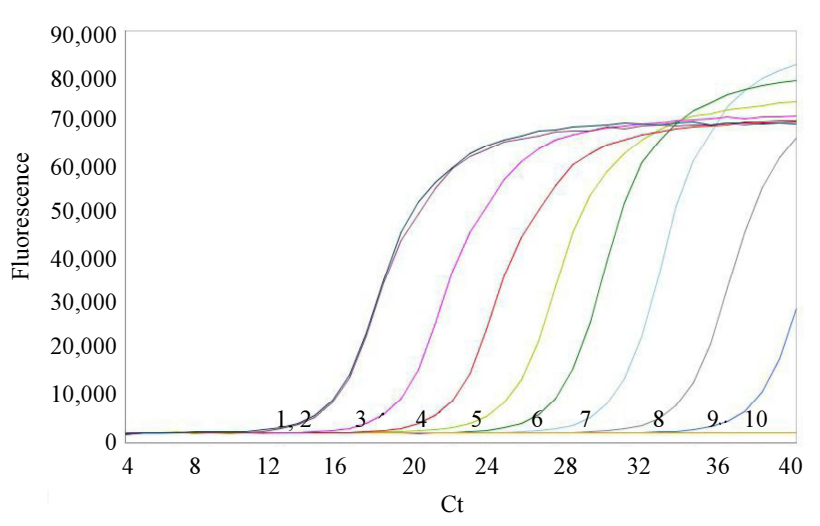

(b)

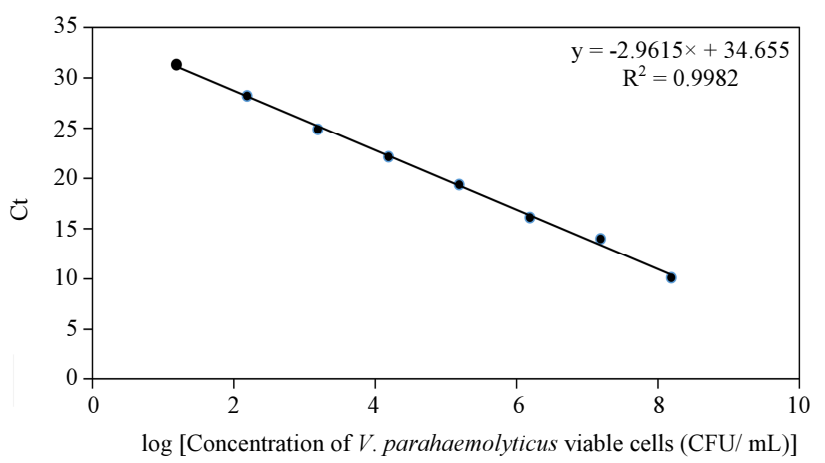

(d)

Fig. 4: Sensitivity of real-time fluorescent PMA-LAMP and PMA-qPCR assay for $V$. parahaemolyticus. The amplification curve of real-time fluorescent PMA-LAMP assay (a) and PMA-qPCR assay (b). The standard curves of the real-time fluorescent PMA-LAMP assay (c) and PMA-qPCR assay (d) were generated from the sample of $V$. parahaemolyticus bacteria solution, ranged from $10^{8}$ to $10^{0} \mathrm{CFU} / \mathrm{mL}$, with the ABI Step One Plus ${ }^{\mathrm{TM}}$. 1-9: $4.5 \times 10^{8}, 4.5 \times 10^{7}, 4.5 \times 10^{6}, 4.5 \times 10^{5}, 4.5 \times 10^{4}, 4.5 \times 10^{3}$, $4.5 \times 10^{2}, 4.5 \times 10^{1}, 4.5 \times 10^{0} \mathrm{CFU} / \mathrm{mL}, 10$ : No template control

Therefore, it confirmed that the real-time fluorescent PMA-LAMP and PMA-qPCR assay for detection of viable $V$. parahaemolyticus cells were highly specific.

\section{Sensitivity of Real-Time Fluorescent PMA-LAMP and PMA-qPCR}

The real-time fluorescent PMA-LAMP and PMA-qPCR detection methods for $V$. parahaemolyticus were established based on PMA treatment. The results showed that the detection limit of real-time fluorescence PMA-LAMP for viable $V$. parahaemolyticus was $4.5 \times 10^{\circ} \mathrm{CFU} / \mathrm{mL}$, which was 10 times higher than that of PMA-qPCR (Fig. 4). The correlation coefficients $\left(\mathrm{R}^{2}\right)$ of the real-time fluorescent PMA-LAMP and PMA-qPCR assays were 0.9992 and 0.9982 , respectively. In addition, the $\mathrm{CV}$ values of initial amplification time and $\mathrm{Ct}$ values of PMALAMP and PMA-qPCR for sensitivity analysis were all lower than $10.0 \%$. It indicated that SYTO-16 dyed real-time fluorescent PMA-LAMP assay had high repeatability and can be used for quantification with high sensitivity.

\section{Artificial Contamination Testing}

Before artificial contamination, all samples purchase from supermarket were confirmed to be $V$. parahaemolyticus negative by BAM methods. As shown in Table 3, three different seafood samples, containing $4.5 \times 10^{5}$ to $4.5 \times 10^{0} \mathrm{CFU} / \mathrm{mL}$ viable $V$. parahaemolyticus ATCC 17082 cells and $4.5 \times 10^{5} \mathrm{CFU} / \mathrm{mL}$ dead cells, were assessed by real-time fluorescent PMA-LAMP, PMA-qPCR and BAM assays (Table 3). The CV values of PMA-LAMP and PMA-qPCR for three different seafood samples were between $0.49 \%$ to $7.88 \%$ and $0.47 \%$ to $4.38 \%$, respectively, which demonstrated high repeatability. Furthermore, the results indicated that $4.5 \times 10^{5} \mathrm{CFU} / \mathrm{mL}$ of the dead cells of $V$. parahaemolyticus did not interfere with the developed real-time fluorescent PMA-LAMP method.

\section{Tests of Practical Samples}

The applicability of fluorescence-quantitative PMALAMP assay for 139 blind samples was demonstrated. 
Table 3: Detection of Vibrio parahaemolyticus PMA-LAMP, PMA-qPCR assays, and BAM in artificially contaminated produce commodities

\begin{tabular}{|c|c|c|c|c|c|c|c|}
\hline \multirow[b]{2}{*}{$\begin{array}{l}\text { Samples } \\
(\mathrm{n}=6 \text { each })\end{array}$} & \multirow[b]{2}{*}{ Strain } & \multirow[b]{2}{*}{$\begin{array}{l}\text { Spiked level } \\
(\mathrm{CFU} / \mathrm{mL})\end{array}$} & \multicolumn{2}{|c|}{$\begin{array}{l}\text { Detection method } \\
\text { PMA-LAMP (min) }\end{array}$} & \multicolumn{3}{|l|}{ PMA-qPCR $(C t)^{a}$} \\
\hline & & & Mean & $\mathrm{CV}(\%)^{\mathrm{b}}$ & Mean & $\mathrm{CV}(\%)^{\mathrm{b}}$ & BAM \\
\hline \multirow[t]{7}{*}{ Shrimp } & $\begin{array}{l}\text { Vibrio } \\
\text { parahaemolyticus }\end{array}$ & $\begin{array}{l}4.5 \times 10^{5} \\
(\text { dead cells })\end{array}$ & - & & - & & - \\
\hline & ATCC 17802 & $4.5 \times 10^{5}$ & $+(16.56 \pm 0.43)$ & 2.6 & $+(19.03 \pm 0.40)$ & 2.1 & + \\
\hline & & $4.5 \times 10^{4}$ & $+(19.68 \pm 1.55)$ & 7.88 & $+(22.94 \pm 0.58)$ & 2.53 & + \\
\hline & & $4.5 \times 10^{3}$ & $+(22.03 \pm 0.28)$ & 1.27 & $+(25.75 \pm 0.12)$ & 0.47 & + \\
\hline & & $4.5 \times 10^{2}$ & $+(25.03 \pm 0.40)$ & 1.6 & $+(29.92 \pm 1.28)$ & 4.38 & + \\
\hline & & $4.5 \times 10^{1}$ & $+(28.94 \pm 0.58)$ & 2.01 & $+(32.63 \pm 0.40)$ & 1.22 & + \\
\hline & & $4.5 \times 10^{0}$ & $+(31.94 \pm 0.18)$ & 0.56 & - & - & + \\
\hline \multirow[t]{7}{*}{ Sleevefish } & & $4.5 \times 10^{5}($ dead cells $)$ & - & & - & & - \\
\hline & & $4.5 \times 10^{5}$ & $+(17.22 \pm 0.21)$ & 1.22 & $+(18.53 \pm 0.41)$ & 2.21 & + \\
\hline & & $4.5 \times 10^{4}$ & $+(19.78 \pm 0.22)$ & 1.11 & $+(22.04 \pm 0.58)$ & 2.63 & + \\
\hline & & $4.5 \times 10^{3}$ & $+(23.03 \pm 0.78)$ & 3.39 & $+(26.75 \pm 0.62)$ & 2.32 & + \\
\hline & & $4.5 \times 10^{2}$ & $+(26.76 \pm 1.18)$ & 4.41 & $+(29.92 \pm 0.88)$ & 2.94 & + \\
\hline & & $4.5 \times 10^{1}$ & $+(30.88 \pm 0.15)$ & 0.49 & $+(32.54 \pm 1.40)$ & 4.3 & + \\
\hline & & $4.5 \times 10^{0}$ & $+(33.22 \pm 0.21)$ & 0.63 & - & - & + \\
\hline \multirow[t]{7}{*}{ Cod } & & $4.5 \times 10^{5}($ dead cells $)$ & - & & - & & - \\
\hline & & $4.5 \times 10^{5}$ & $+(17.76 \pm 0.99)$ & 5.56 & $+(19.55 \pm 0.81)$ & 4.14 & + \\
\hline & & $4.5 \times 10^{4}$ & $+(20.62 \pm 0.12)$ & 0.58 & $+(22.24 \pm 0.85)$ & 3.82 & + \\
\hline & & $4.5 \times 10^{3}$ & $+(24.19 \pm 0.35)$ & 1.45 & $+(24.75 \pm 0.26)$ & 1.05 & + \\
\hline & & $4.5 \times 10^{2}$ & $+(27.03 \pm 0.63)$ & 2.33 & $+(27.92 \pm 0.88)$ & 3.14 & + \\
\hline & & $4.5 \times 10^{1}$ & $+(30.67 \pm 0.64)$ & 2.09 & $+(31.54 \pm 0.74)$ & 2.35 & + \\
\hline & & $4.5 \times 10^{0}$ & $+(33.38 \pm 1.73)$ & 5.18 & - & - & + \\
\hline
\end{tabular}

$\mathrm{C}_{\mathrm{t}}^{\mathrm{a}}$ values are given where the fluorescence signal was higher than the background.

$\mathrm{CV}(\%)^{\mathrm{b}}$ are Coefficient of variation.

+ , Vibrio parahaemolyticus positive by the method. -, Vibrio parahaemolyticus negative by the method

Table 4: Detection of practical samples by real-time fluorescent PMA-LAMP assay compared with PMA-qPCR and BAM method

\begin{tabular}{|c|c|c|c|c|}
\hline \multirow[b]{2}{*}{ Samples } & \multirow[b]{2}{*}{$\begin{array}{l}\text { Number } \\
\text { of samples }\end{array}$} & \multicolumn{3}{|c|}{ Positive number } \\
\hline & & PMA-LAMP & PMA-qPCR & BAM method \\
\hline Shrimp $^{a}$ & 33 & 1 & 1 & 1 \\
\hline Sleeve-fish ${ }^{\mathrm{a}}$ & 12 & 0 & 0 & 0 \\
\hline Trichiurus lepturus $^{\mathrm{a}}$ & 11 & 0 & 0 & 0 \\
\hline $\operatorname{Cod}^{\mathrm{a}}$ & 14 & 1 & 1 & 1 \\
\hline Meretrix $^{a}$ & 12 & 0 & 0 & 0 \\
\hline Grilled fish $^{\mathrm{a}}$ & 18 & 0 & 0 & 0 \\
\hline Grilled yellow croaker ${ }^{\mathrm{a}}$ & 10 & 1 & 1 & 1 \\
\hline Dried squid $^{\mathrm{a}}$ & 12 & 0 & 0 & 0 \\
\hline Dried squid $^{b}$ & 17 & 0 & 0 & 0 \\
\hline Total & 139 & 3 & 3 & 3 \\
\hline Positive detection rate $(\%)$ & - & 2.16 & 2.16 & 2.16 \\
\hline
\end{tabular}

${ }^{\mathrm{a}}$ Purchased from local market.

${ }^{\mathrm{b}}$ Afforded by Zhoushan Entry-Exit Inspection and Quarantine Bureau

All the samples were also analyzed by PMA-qPCR assay and BAM method. The results showed that two of the above three methods were detected in 139 samples, with a detection rate of $2.16 \%$. The concordance between real-time fluorescent PMALAMP and PMA-qPCR tests in 139 blind samples for detection of the $V$. parahaemolyticus were shown (Table 4). The results of the two methods are consistent with the rate of $100 \%$.

\section{Discussion}

V. parahaemolyticus has been reported to be the causative agent in $50-70 \%$ from all prevalent food poisoning outbreaks as a widely distributed halophile pathogenic in coastal and estuarine environments (Wang et al., 2012; Ye et al., 2014). In the past decades, there were many several reliable and powerful technologies available for the detection of $V$. 
parahaemolyticus genomic DNA (Yang et al., 2018). The most widely used method for detection of $V$. parahaemolyticus infection was based on the molecular level, such as polymerase chain reaction (PCR). However, due to the need for complex instruments, complex laboratory procedures, expensive reagents and trained personnel, this method may not be suitable for use in developing countries or in the field (Chen and Ge, 2010; Li et al., 2017). Therefore, an ever-expanding array of novel nucleic acid amplification techniques have been developed to meet the challenge of performing diagnostics without well-equipped equipment in lowresource environments (Fang et al., 2018). On this basis, a variety of nucleic acid detection methods were developed under isothermal conditions. Some of these methods have been shown to detect small amounts of nucleic acid copies (Kanayama et al., 2008; Li et al., 2018; Peng et al., 2018). Among these isothermal methods, LAMP assay is an excellent diagnostic tool. It offers an attractive option because it is simple, cost effective, efficient and specific. In isothermal conditions, the test can be performed using a water bath or a heating block, which are readily available under electrified conditions (Cao et al., 2010).

Although this method has significant specificity, its disadvantage is that once the test tube is opened, there will be contamination after culture. In order to reduce the risk of pollution and eliminate the need to open the tubes, the fluorescence dyes offer greater flexibility and less cost. Fluorescence-based detection helps prevent false negatives that may result from base pair mismatches when template sequences change (Seyrig et al., 2015). As long as the optimum concentration of fluorescent dye is determined, the method gave the best results of short initial amplification time, high fluorescence intensity, low inhibition rate and best effect. Signal-to-noise ratio is an important characteristic of the ratio of signal intensity to noise intensity. High SNR means high reliability of signal and low probability of false positive result. In previous studies, the signal-to-noise ratio was calculated by comparing the reaction performed by DNA template with the fluorescence ratio of the non-template control (Oscorbin et al., 2016). According to the rule, SYTO-16 demonstrated the best SNR at appropriate concentrations tested and gave better results than other fluorescence dyes.

Traditional LAMP detection method could not distinguish the dead and viable cells of $V$. parahaemolyticus, while PMA-LAMP and EMA -LAMP method made up this defect. It has been reported that the toxicity of PMA dyes is far less than that of EMA dyes. Under the same conditions, the working concentration of PMA is always higher than that of EMA (Nagamine et al., 2002). Therefore, PMA-based LAMP method has more advantages in the rapid detection of $V$. parahaemolyticus. Zhong et al. (2016) employed SYTO-9 dye to develop a
PMA-based LAMP for rapid detection of $V$. parahaemolyticus. The amplification was confirmed in 7 min (7cycles) and the detection limit was $6.8 \times 10^{\circ}$ copies.

In this study, the PMA-based LAMP method was established by using a fluorescent dye SYTO-16 with higher SNR and amplification efficiency for the detection of $V$. parahaemolyticus. It was simple and effective for specific detection of the toxR gene under optimized conditions at 65 ${ }^{\circ} \mathrm{C}$ for $40 \mathrm{~min}$. The initial amplification time was defined as the moment of produced fluorescence. Based on this, the amplification was observed from $5 \mathrm{~min}$, the complete reaction duration was optimized to $40 \mathrm{~min}$. The fluorescence dye concentration was optimized because these can affect the previously described signal-to-noise ratio. The results showed that $10 \mu \mathrm{M}$ Syto 16 was the best combination of time-threshold and SNR in LAMP reaction. In addition, betaine did not affect the amplification of the short target fragment $(<300 \mathrm{bp})$ and reduced the nonspecific amplification (Suebsing et al., 2013).

The PMA-LAMP method was able to detect $V$. parahaemolyticus in as little as $4.5 \times 10^{\circ} \mathrm{CFU} / \mathrm{mL}$ DNA template extracted from infected seafood, which was consistent with the results of PMA-qPCR. Meanwhile, the number of genomes in the PMALAMP method was linearly correlated with the initial amplification time. In these experiments, the time required to form a visual fluorescence value unit is inversely proportional to the number of templates, indicating that the number of signals is proportional to the abundance of templates (Burbulis et al., 2015). The result of the standard curves indicated that there were significant correlations between the initial amplification time and the template concentration $\left(\mathrm{R}^{2}=0.9992\right)$. In addition, no cross-amplification with other pathogens was found, indicating that the assay was highly specific for the detection of $V$. parahaemolyticus. The applicability of the fluorescence-quantitative LAMP assay was assessed with field samples and the results were compared with those obtained from the qPCR method. It showed that in 139 blind samples, the positive detection rates were the same $(2.16 \%)$ by using the fluorescencequantitative PMA-LAMP and PMA-qPCR, which were in agreement with the BAM method. The results of the two methods are consistent with each other. This method, combined with a simple and rapid DNA extraction method, can be used for the identification of $V$. parahaemolyticus live cells in field and laboratory, saving time and cost. However, this developed method should be applied and validated by using more natural seafood samples in the future.

The LAMP assay was capable of amplifying a DNA target under isothermal conditions and the amplification products can be quantitatively detected. In addition, PMA- 
based LAMP method made up for the inability to identify the dead and live cells. The assay could be performed in less than $40 \mathrm{~min}$ without special equipment. Compared with PMA-qPCR method, it has greater advantages in sensitivity and practical sample detection. In conclusion, this method provides great advantages for the rapid field diagnostics of $V$. parahaemolyticus infection in aquatic products. It can be considered as a powerful technology for the monitoring/tracing of $V$. parahaemolyticus, which was rapid, accurate, simple and economic, with good sensitivity and specificity.

\section{Conclusion}

The LAMP assay was capable of amplifying a DNA target under isothermal conditions and the amplification products can be quantitatively detected. In addition, PMAbased LAMP method made up for the inability to identify the dead and live cells. The assay could be performed in less than 40 min without special equipment. Compared with PMA-qPCR method, it has greater advantages in sensitivity and practical sample detection. In conclusion, this method provides great advantages for the rapid field diagnostics of $V$. parahaemolyticus infection in aquatic products. It can be considered as a powerful technology for the monitoring/tracing of V. parahaemolyticus, which was rapid, accurate, simple and economic, with good sensitivity and specificity.

\section{Acknowledgements}

We thank the researchers who facilitated the completion of this study and we are also grateful to Zhoushan Entry-exit Inspection and Quarantine Bureau for providing samples.

\section{Funding Information}

The work was supported by the National Key Research and Development Program of China (2017YFF0210200, 2018YFF0215205), the Public Projects of Zhejiang Province (LGC19C200006).

\section{Author's Contributions}

Mingzhou Zhang: Has guided the project, determined the research framework, data analysis and involved in paper writing

Huanteng Yu: Has contributed to the experimental works, data analysis and paper writing

Jiehong Fang: Has contributed in the data analysis and paper writing

Biao Ma: Has contributed in the data analysis and paper writing

Jiali Li: Has contributed in the experimental works and data analysis

\section{Ethics}

This article does not contain any studies with human participants or animals performed by any of the authors.

\section{References}

Nair, G.B., T. Ramamurthy, S.K. Bhattacharya and B. Dutta, 2007. Global dissemination of Vibrio parahaemolyticus serotype $\mathrm{O} 3: \mathrm{K} 6$ and its serovariants. Clinical Microbiol. Reviews, 20: 39-48. DOI: 10.1128/CMR.00025-06

Guo, Z., Y. Jia, X. Song and J. Lu, 2010. Giant gold nanowire vesicles based colorimetric and SERS dual mode immunosensor for ultrasensitive detection of Vibrio parahemolyticus. Analytical Chem., 90: 61246130. DOI: 10.1021 acs.analchem.8b00292

Su, Y. and C. Liu, 2007. Vibrio parahaemolyticus: A concern of seafood safety. Food Microbiol., 24: 549-558. DOI: 10.1016/j.fm.2007.01.005

Zhong, Q., J. Tian, B. Wang and W. Li, 2016. PMA based real-time fluorescent LAMP for detection of Vibrio parahaemolyticus in viable but nonculturable state. Food Control, 63: 230-238. DOI: 10.1016/j.foodcont.2015.11.043

Stalin, N. and P. Srinivasan, 2016. Characterization of Vibrio parahaemolyticus and its specific phage from shrimp pond in Palk Strait, South East coast of India. Biol., 44: 526-533. DOI: $10.1016 /$ j.biologicals.2016.08.003

Zhi, A., B. Ma, Y. Wu and J. Fang, 2017. Detection of viable Vibrio cholerae cells in seafood using a real-time visual loop-mediated isothermal amplification combined with propidium monoazide. Food Analytical Methods, 11: 99-110. DOI: $10.1007 / \mathrm{s} 12161-017-0981-4$

Davis, C.R., L.C. Heller, K.K. Peak and D.L. Wingfield, 2004. Real-time PCR detection of the thermostable direct hemolysin and thermolabile hemolysin genes in a Vibrio parahaemolyticus cultured from mussels and mussel homogenate associated with a foodborne outbreak. J. Food Protection, 67: 1005-1008. DOI: $10.4315 / 0362-028 X-67.5 .1005$

Cai, T., L. Jiang, C. Yang and K. Huang, 2006. Application of real-time PCR for quantitative detection of Vibrio parahaemolyticus from seafood in eastern China. FEMS Immunol. Med. Microbiol., 46: $180-186$. DOI: $10.1111 / \mathrm{j} .1574-695 X .2005 .00016 . x$

Bunpa, S., M. Nishibuchi, J. Thawonsuwan and N. Sermwittayawong, 2018. Genetic heterogeneity among Vibrio alginolyticus strains and design of a PCR-based identification method using gyr B gene sequence. Canadian J. Microbiol., 64: 1-10. DOI: $10.1139 /$ cjm-2017-0269 
Xu, D., L. Ji, X. Wu and W. Yan, 2018. Detection and differentiation of Vibrio parahaemolyticus by multiplexed real-time PCR. Canadian J. Microbiol. , 64: 809-815. DOI: 10.1139/cjm-2018-0083

Notomi, T., H. Okayama, H. Masubuchi and T. Yonekawa, 2000. Loop-mediated isothermal amplification of DNA. Nucleic Acids Res., 28: 63-63. DOI: 10.1097/RLU.0b013e3181f49ac7

Nagamine, K., T. Hase and T. Notomi, 2002. Accelerated reaction by loop-mediated isothermal amplification using loop primers. Molecular Cellular Probes, 16: 223-229. DOI: 10.1006/mcpr.2002.0415

Oscorbin, I.P., E.A. Belousova, A.I. Zakabunin and U.A. Boyarskikh, 2016. Comparison of fluorescent intercalating dyes for quantitative loop-mediated isothermal amplification (qLAMP). Biotechniques, 61: 20-25. DOI: 10.2144/000114432. PMID: 27401670

Udovich, J.A., D.G. Besselsen and A. F. Gmitro, 2010. Assessment of acridine orange and SYTO 16 for in vivo imaging of the peritoneal tissues in mice. J. Microscopy, 234: 124-129. DOI: 10.1111/j.1365-2818

Wang, L. and A. Mustapha, 2010. EMA-real-time PCR as a reliable method for detection of viable Salmonella in chicken and eggs. J. Food Sci., 75: 134-139. DOI: $10.1111 / \mathrm{j} .1750-3841.2010 .01525 . \mathrm{x}$

Zhu, R., T. Li, Y. Jia and L. Song, 2012. Quantitative study of viable Vibrio parahaemolyticus cells in raw seafood using propidium monoazide in combination with quantitative PCR. J. microbial. methods, 90: 262-266. DOI: 10.1016/j.mimet.2012.05.019

Saiyudthong, S. and S. Trevanich, 2013. An optimized EMA-RAPD-PCR for a reliable detection of viable Salmonella spp. in chicken products. J. Food Safety, 33: 247-258. DOI: $10.1111 /$ jfs. 12046

Fittipaldi, M., A. Nocker and F. Codony, 2012. Progress in understanding preferential detection of live cells using viability dyes in combination with DNA amplification. J. microbial. methods, 91: 276-289. DOI: 10.1016/j.mimet.2012.08.007

Fang, J., Y. Wu, D. Qu and B. Ma, 2018. Propidium monoazide real-time loop-mediated isothermal amplification for specific visualization of viable Salmonella in food. Letters applied microbial., 67: 79-88. DOI: 10.1111/lam.12992

Wang, L., Y. Li, J. Chu and Z. Xu, 2012. Development and application of a simple loop-mediated isothermal amplification method on rapid detection of Listeria monocytogenes strains. Molecular Biol. Reports, 39: 445-449.

DOI: $10.1007 / \mathrm{s} 11033-011-0757-7$

Ye, Y., H. Li, Q. Wu and L. Na, 2014. Immunocaptured loop mediated isothermal amplification assay for detection of Vibrio parahaemolyticus in seafood. J. Food Safety, 34: 21-25. DOI: $10.1111 /$ jfs. 12090
Yang, H., S. Wei, R. Gooneratne and A.N. Mutukumira, 2018. Development of a recombinase polymerase amplification assay for Vibrio parahaemolyticus detection with an internal amplification control. Canadian J. Microbiol., 64: 223-230.

DOI: $10.11139 / \mathrm{cjm}-2017-0504$

Chen, S. and B. Ge, 2010. Development of a toxR-based loop-mediated isothermal amplification assay for detecting Vibrio parahaemolyticus. BMC Microbiol., 10: 1-9. DOI: 10.1186/1471-2180-10-41

Li, Y., P. Fan, S. Zhou and L. Zhang, 2017. Loopmediated isothermal amplification (LAMP): A novel rapid detection platform for pathogens. Microbial pathogenesis, 107: 54-61. DOI: $10.1016 /$ j.micpath.2017.03.016

Kanayama, A., E. Fujihara, T. Saika and I. Kobayashi, 2008. Detection of Chlamydia trachomatis and Neisseria gonorrhoeae in urine samples of males and females by the strand displacement amplification (SDA) method. Kansenshogaku Zasshi, 82: 182-186. DOI: 10.11150/kansenshogakuzasshi1970.82.182

Li, Z., Y. Xiong, P. Zhu and H. Huang, 2018. Sensitive and rapid detection of sulfate-reducing bacteria in jet fuel by loop-mediated isothermal amplification combined with lateral flow dipstick. Am. J. Biochem. Biotechnol., 14: 117-123.

DOI: $10.3844 /$ ajbbsp.2018.117.123

Peng, Z., W. Gao, H. Huang and J. Jiang, 2018. Rapid detection of Vibrio parahaemolyticus in shellfish by real-time recombinase polymerase amplification. Food Analytical Methods, 11: 2076-2084. DOI: $10.1007 / \mathrm{s} 12161-018-1188-\mathrm{Z}$

Cao, Y.T., Z.H. Wu, J.C. Jian and Y.S. Lu, 2010. Evaluation of a loop-mediated isothermal amplification method for the rapid detection of Vibrio harveyi in cultured marine shellfish. Letters applied microbial., 51: 24-29. DOI: 10.1111/j.1472-765X.2010.02853.x

Seyrig, G., R.D. Stedtfeld, D.M. Tourlousse and F. Ahmad, 2015. Selection of fluorescent DNA dyes for real-time LAMP with portable and simple optics. J. Microbial. Methods, 119: 223-227.

DOI: 10.1016/j.mimet.2015.11.004

Suebsing, R., P. Prombun, J. Srisala and W. Kiatpathomchai, 2013. Loop-mediated isothermal amplification combined with colorimetric nanogold for detection of the microsporidian Enterocytozoon hepatopenaei in penaeid shrimp. J. applied microbial., 114: 1254-1263. DOI: 10.1111/jam. 12160

Burbulis, I.E., Y. Kumiko, O.V. Nikolskaia and S.T. Prigge, 2015. Detection of pathogen-specific antibodies by loop-mediated isothermal amplification. Clinical Vaccine Immunol., 22: 374-380.

DOI: $10.1128 /$ CVI.00811-14 